\title{
Three Hierarchy Map Building Based on Artificial Label
}

\author{
Wu Hao ${ }^{1}$, Tian Guohui ${ }^{1, *}$, Pan Chunwei ${ }^{2,3}$, Wang Xin ${ }^{1}$ and Yu Jinshan ${ }^{1}$ \\ ${ }^{1}$ Department of Control Science and Engineering, University of Shandong, Jinan, Shandong 250061, China; ${ }^{2}$ School of \\ Information and Electric Engineering, Shandong Jianzhu University, Jinan 250101, China; ${ }^{3}$ Shandong Provincial Key \\ Laboratory of Intelligent Buildings Technology, Shandong Jianzhu University, Jinan 250101, China
}

\begin{abstract}
When service robots carry on mission planning and execution, they not only need structural information about the environment to navigation and localization, but also need to possess deeper knowledge to endow a robot with higher degrees of autonomy and intelligence. However it is hard for a robot to cognize the semantic description such as the function attributes of objects and rooms only relying on robot's sensors itself. When the exploration scope of the robot is expanded to large regions such as a building, the problem becomes more prominent. In this paper, artificial labels are used to provide semantic concepts and relations of objects and rooms in a building, which can solve the complexity and limitations of semantic recognition and scene understanding only with robot's vision. Imitating spatial cognizing mechanism of human, three hierarchy space model is built for a building service robot. With this space model, not only the plane structure of the environment for navigation is known, but also the abundant semantic information in different kinds of environment are obtained. Simulation results show that the three hierarchy indoor space model is applicable to service tasks of indoor robot, understanding human semantic statement, produce reasonable service path, and ensuring the security of robot running in building environment.
\end{abstract}

Keywords: Artificial label, hierarchy model, intelligence space, semantic map.

\section{INTRODUCTION}

The emergences of service robots in our work and life bring us lots of conveniences more than ever before. To perform these tasks, the robot should move freely in varied scenes of a building such as corridors, elevator, living room and printing room. Thereby, the moving scope of the robot becomes larger and larger. Aiming to large scale unknown environment, the study of SLAM has become the hotspot of current research for map building. Many in-depth researches have been done to solve data association, path exploring, identification of static and moving objects in dynamic environment, and understanding \& extraction of the environmental characteristics, etc. However, due to the restriction of the robot's perceptual ability, some key problems are still unsolved that restrict map's application to robot navigation [1-5].

Semantic map is illumed by human's task performing steps. Semantic map should include detailed information about the function property of rooms and objects $[6,7]$. The locations of objects can show the semantic functions. For example, there must have printer in printing room. Thus using the location information of objects, semantic path planning and navigation can be realized. Identifying large objects and doors are important to build semantic maps.

High-level semantic feature extraction algorithm is used to identify the objects and the doors, which are regarded as a partition warranty to form a global topological map, and the object relationships in the room are used to form object associated map which is treated as a local map. However, the morphological changes of the door, different visual angle, illumination change, shelter and other matters will affect the accuracy of recognition $[8,9]$. The problems mentioned above result in unsatisfactory identified result, and functional properties of objects are difficult to be obtained relying solely on robot vision [8-10]. Illumination change, viewing angles, shelter and other issues also affect its recognition accuracy. And the operation method, function and other properties of the objects are impossible to be obtained only by visual observations. As the moving scope of the robot is becoming larger and larger, such as a building, the process of semantic map building is more and more difficult. Combining with layered map building idea, using QR code based artificial labels, three hierarchy space model is a significative attempt to easily build semantic map for a building service robot.

\section{THREE HIERARCHY SPACE MODEL AND THE DESIGN OF ARTIFICIAL LABELS}

\subsection{Three Hierarchy Space Model Applying to Building Service}

In order to make a service robot be intelligent, we should get inspired from the representation method of the space environment used by people, and study the spatial representation of service robots to relate with human language information. Human's understanding to the environment is carried out at different levels: the concepts of storey and corridors are constructed for the large space; apartments and rooms are formatted for the small space; the objects operated by robots are located at somewhere of a room, which is 


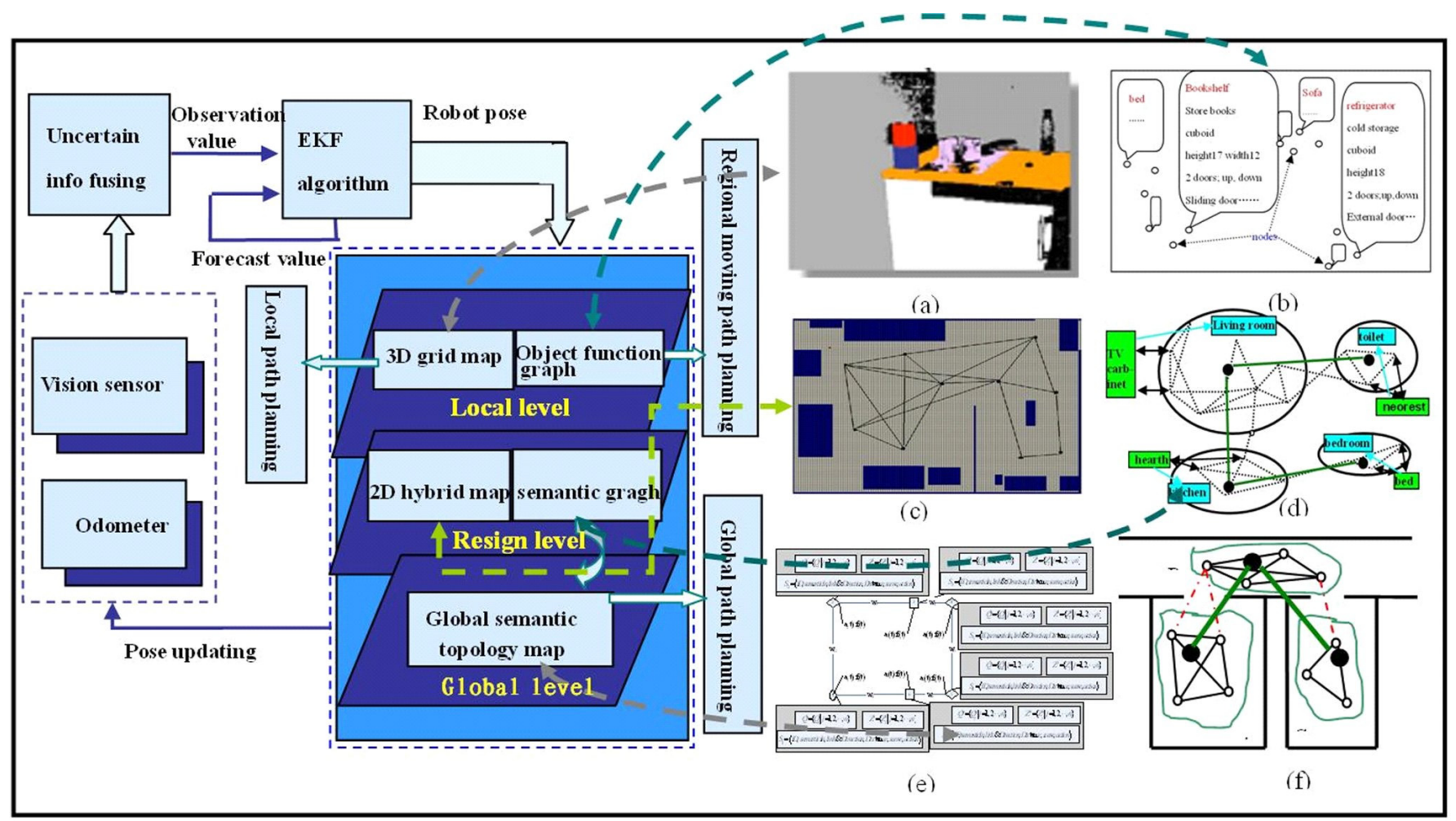

Fig. (1). The structure of three hierarchy space model.

looked as local smaller area. The corners and doorplates are key features which use as navigation basis to the people; the placement of large items in unit room and the relations between objects and rooms can provide human the basis of region path planning; the searching and grasping of the objects in local complex space can be based on threedimensional space model around the object receiving from human's eyes. Human uses three level cognizing methods to remember the environment from macroscopic to microscopic view, from broad categorizing style to the details.

Therefore, the robot can imitate spatial modeling method of the human and build indoor three hierarchy environment maps in unknown environment. During the map building, the semantic information is added to describe the function property and ascription relation of objects, rooms, corridors and floors. According to the service features of the building, the three hierarchy space model is built which is shown as Fig. (1).

\subsection{The design of artificial signpost label and flat label}

QR code (Quick Response code) technology coming from Denso Corporation of Japan is the most representative two- dimensional bar code currently. In addition to the advantage of large capacity, high reliability, strong security and confidentiality, its recognition orientation can reach 360 degrees, and it is effective in representation of character [10, $11]$.

The semantic describing information of the environment is transformed to data encoding, then QR code is formed. QR code based labels are mainly pasted at three kinds of positions in three-level indoor map model: the key positions in corridor area; the doors of flatlet; the large objects in rooms. The first is called artificial signpost label, which mainly provides the semantic information of navigation and context description in large scale environment; the second is called artificial flat label, which mainly provides the structure information of the suites; the third is called artificial object label, which mainly provides the information of large objects such as name, shape, function and attribute.

Signpost label and flat label are all used in corridor. Corridor is a typical structured environment that has single hues, dominated by cold hues. To identify the artificial label quickly from long distance and estimate the relative location of the robot and the artificial label, color and graph based artificial signpost label and flat label are designed, shown as Figs. (2a and $\mathbf{2 b}$ ).

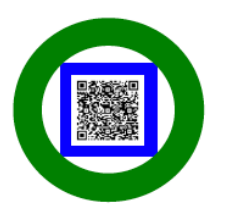

(a) signpost label

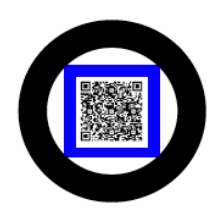

(b) flat label

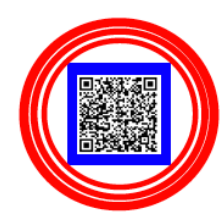

(c) object label
Fig. (2). QR code based artificial labels.

The label is made up of two parts: periphery mode and the inside information. The artificial object label's periphery is named as mode part which includes colored rings and blue rectangular; and the insider is named as information part which includes QR code. The coding of signpost label mainly stores the semantic info of current location, area structure info, the function semantic info and context info for navigation. The coding of flat label mainly stores the structure info of the room. The periphery modes of signpost and flat label are similar, made up of one color circle and a blue 
box inside. The color of the circle is decided by the pasted location of the label. The structure in the flatlet is more complex than that in corridor. There are many kinds of objects, and the artificial labels pasted on large objects are often shaded. The detailed realization method of the artificial label recognition and closing refers to the thesis [11].

\section{THREE HIERARCHY MAP BUILDING STRATEGY BASED ON THE FEATURE OF DIFFERENT ENVI- RONMENT}

\subsection{Global Semantic Topology Map Building based on Distributed Information Representation}

Floors and corridors are structuring environment with a spot of typical features, so building topology map including nodes coordinate information is traditional mapping method. It can reflect the positions and relations of nodes. However, when the physical position of the node is uncertain, it is difficult to achieve the robot's exact navigation, which must reduce robot autonomy. According to the signpost navigation patterns of the human, the artificial signpost labels based on QR code technology are distributed in the key positions of the environment to form cognitive-oriented points. It can not only give semantic information of navigation, but also provide the semantic characteristic of the environment with respect to mapping. Based on the fusing localization technology of intelligent space, the global fuzzy topology semantic map with "distributed information representation" is formed. The map has three specialties: global, semantic, and topological, which are showed in Figs. (1e and 1f).

At the cognitive-oriented point in the semi-unknown environment, the robot decides its moving direction based on the artificial labels and realizes navigation. At the same time, the robot builds a time-space network which can reflect time and space relationship between nodes. Consider the robot itself as a centre to establish a topological neighborhood around the current node position. That likes the biological nervous system in which excited neuron makes the surrounding neurons excite together. The relation weight between the current node and the neighborhood node will update with it. Immune network algorithmis used to form the environmental awareness mechanism of "distributed representation", then the global semantic topology map is built.

\subsubsection{Time-Sequential Memory Mechanism of Network Nodes}

Immune cells in the network have self-attenuation. That is, if the external input doesn't stimulate it, the cell's activities will gradually decrease. The vitality of immune cell is defined as below:

$$
a_{i}(t)=\left\{\begin{array}{cc}
0 & \forall a_{j}(t)=1 \\
1 & f_{i}(\mathrm{t})=1 \& a_{i}^{C}(t-1)=0 . \\
\eta a_{i}(t-1) & \text { else }
\end{array}\right.
$$

$f_{i}(t)=1$ means the scene tag of the node $i$ at time $t$ is found. The vitality of antibody reflects the distance between nodes.

\subsubsection{Growth Mechanism of Immune Cells}

The emergence of each new sequence will produce a new immune cell and activate the immune cells. At the same time a connection with an existing active cell is brought. The connection weight $w_{i j}$ will reflect when the foregoing antibody $j$ give maximum impact on cell $i$.

$\omega_{i j}(t)=\left\{\begin{array}{cc}0 & t=0 \\ k a_{i}(t) a_{j}(t-1) & a_{j}(t)=0 \& a_{i}(t)=1 . \\ \omega_{i j}(t-1) & \text { else }\end{array}\right.$

$a_{j}(t)$ is the activity of foregoing antibody $j$ (output), $a_{i}(t)$ is the activity of the antibody $i . k$ is learning regulation rate. If the output of the foregoing antibody is larger, antibody activity will be stronger, and the connection weights will be stronger too.

\subsection{Regional Semantic Planning Map Building based on QR Code Technology}

When a robot moves into a suite, it will build 2D hybrid map of the suite. As the robot works in the room, it will find some artificial object labels pasted on large objects.

The name, shape, color, size, functionality and other attribute information of large objects have been written in QR code. The robot reads QR code in the observed image, and adds the information into the object function database immediately. Simultaneously, the correlation between observation position (node) and the corresponding object is established, the relations of objects and rooms are formulated, which are supplemented to object relation database. Object function database and relation database are combined to object information database, which is the fundamental guarantee for management of household goods.

When the regional topology map based on flat labels is established, the nodes(the positions where the robot observes the objects.) in the same room will form a set that corresponds to the concept of room. The four blue clouds in Fig. (3) represent four certain room areas. Using the established attributive relation between observation positions (nodes) and object, the object can be attributable to the room associated with the nodes. That is, as the function of items is known, the room's function can be determined according to the object's function. Thereby the robot can know the attributive relation between rooms and objects. Shown as Fig. (3), the robot recognizes the bookcase which belongs to room $2^{\#}$. Thinking of the bookcase's function, the robot can conclude that the function of room $2^{\#}$ is a study. By the same token, room $1^{\#}, 3^{\#}$ and $4^{\#}$ are named as kitchen, bedroom and living room respectively. Then function semantic topology map is got which not only contains topology relation between rooms, but also includes semantic description of object function, objects adscription and relation, and room functions.

The set of object information database is: $S_{P}=\left\{I_{1}, I_{2}, \cdots, I_{n}, \cdots\right\} \circ$ In that,

$I_{n}=\left\{v \oplus g \oplus w \mid v \in V, g \in G_{N}, w \in W\right\}$. 


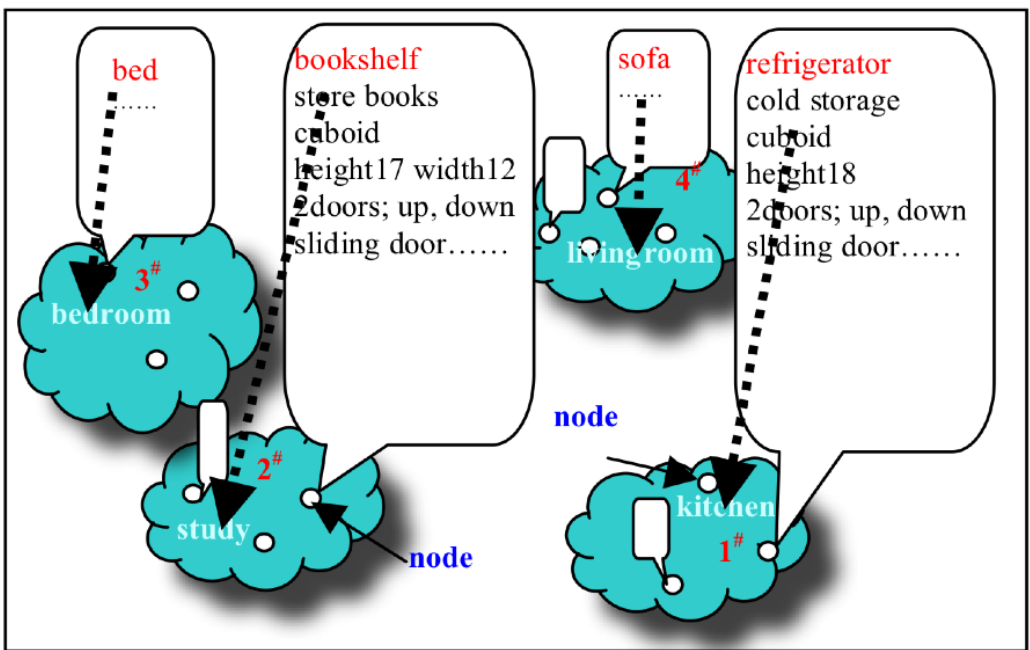

Fig. (3). The diagrammatic sketch of a regional semantic map.

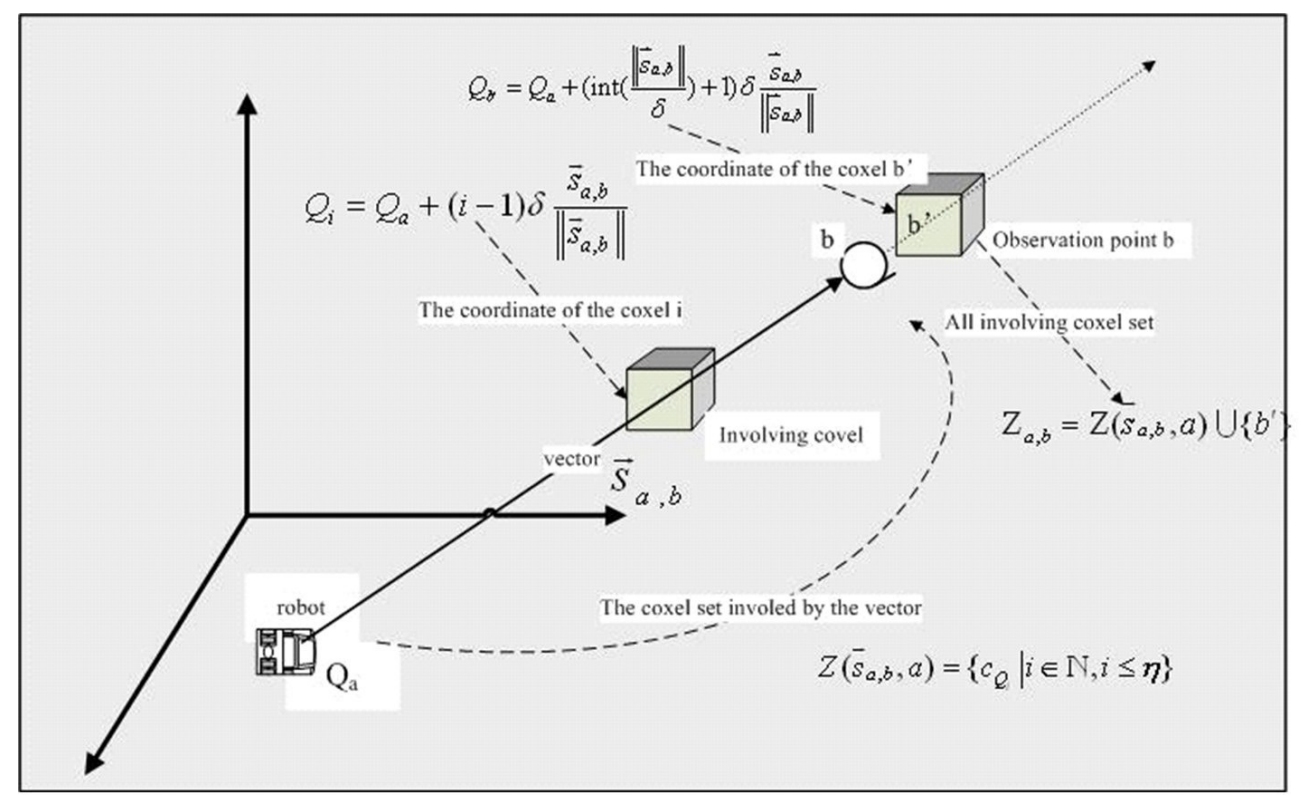

Fig. (4). The relation of the robot and observation position.

$I_{n}$ is the set of object information, $V$ is the set of observation nodes in the environment, $G_{N}$ is the set of object attributes, $W$ is the set of divided rooms; $v$ is the specific node corresponding to the object, $g$ is the specific property of the object, $w$ is the attributive room where the object exists, $" \oplus$ " represents correlation.

\subsection{The Local 3D Grid Map Building based on Occupy Probability Model}

As the appearance of large numbers of humanoid robots and more complex indoor structure, the simple 2D information can't give general description for the surrounding. If the robot imitates human's object searching mode in local area to build 3D map for complicated environment, as shown in Fig. (1a), the robot's task completion can be satisfied.

Using binocular vision schlepped on the robot, the depth information of the local small space are got by SIFT algorithm, and the uncertain mathematic models of binocular vision information are structured based on DSmT evidence theory.

The indoor environment is defined as 3-D grid space which set is $\mathrm{K}$. Each grid cell is called as voxel $c_{x, y, z} . \mathrm{K}$ is the set of all voxels.

The definition of Voxel: in the true 3D space of geography information system, it is the smallest volume cell with fixed size which is used to describe the data memory, management and expression. Voxel is a cube with side length $\delta$.

$o e\left(c_{x, y, z}\right)$ is defined as O\&E value which describes the coverage degree of each voxel with the value range as $[0,1]$. The value of $o e\left(c_{x, y, z}\right)$ means three kinds of coverage condition: occupied, empty and unknown.

Suppose the robot is located at the dot ' $a$ ' currently and the coordinate is $Q_{a}$, which is shown as Fig. (4). The position 


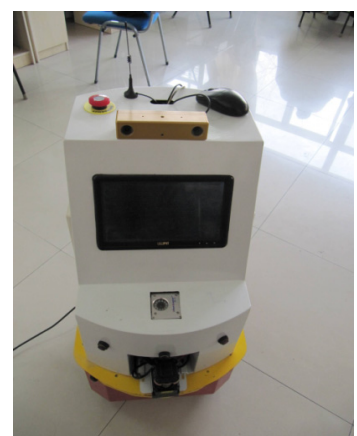

(a)

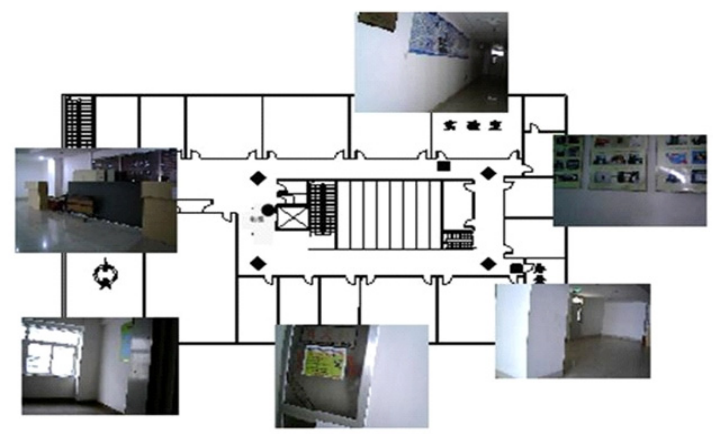

(b)

Fig. (5). Leader 1-DX robot.

of the matching feature point $o_{j}$ is described as $b$ in Fig. (4) which is observed at $a$. The line linking from $\mathrm{a}$ to $\mathrm{b}$ forms the vector $\vec{s}_{a, b} \in \mathrm{P}^{3}$. The O\&E probability values of the voxels drilled through by the vector $\vec{s}_{a, b}$ will be changed during this observation. The set of the voxels which are drilled through can be defined as formation (4):

$Z\left(\vec{s}_{a, b}, a\right)=\left\{c_{Q_{i}} \mid i \in \mathrm{N}, i \leq \eta\right\}$.

In formation (5), $\eta$ means the numbers of the voxels being drilled through. $Q_{i}$ means the coordinate of the $i_{-}$th voxel which is drilled through.

$Q_{i}=Q_{a}+(i-1) \delta \frac{\vec{s}_{a, b}}{\left\|\vec{S}_{a, b}\right\|}$.

$\eta=\operatorname{int}\left(\frac{\left\|\vec{S}_{a, b}\right\|}{\delta}\right)+1$.

Thinking of the voxel $b^{\prime}$ behind the obstacle position $\mathrm{b}$, it should be confirmed as occupying state, so the formation (7) is got.

$Q_{b^{\prime}}=Q_{a}+\left(\operatorname{int}\left(\frac{\left\|\vec{s}_{a, b}\right\|}{\delta}\right)+1\right) \delta \frac{\vec{s}_{a, b}}{\left\|\vec{s}_{a, b}\right\|}$.

Then the set of voxels, effected from the robot position $a$ to the feature point $b$, is defined as below.

$\mathrm{Z}_{a, b}=\mathrm{Z}\left(\vec{s}_{a, b}, a\right) \bigcup\left\{b^{\prime}\right\}$.

Owing to the influence of unknown environmental factors and the limitations of robot body sensors, it is hard to eliminate the uncertainty of the perceptive information. Therefore, when the robot perceives the environment each time, in addition to modeling for the uncertain information, we need to fuse the information perceived by the robot. DSmT algorithm can be used to fuse the imprecise and high contradictory information statically or dynamically.

The reliability, the decision-making rules and combination rules in DSmT are based on super-power set space. Set two focal elements $\theta_{1}$ and $\theta_{2}$ in the identification box@. The circumscription $\theta_{1}$ means that voxel is occupied and $\theta_{2}$ means that voxel is empty. Its super-power set space is $D^{\Theta}=\left\{\phi, \theta_{1}, \theta_{2}, \theta_{1} \cap \theta_{2}, \theta_{1} \cup \theta_{2}\right\} . \phi$ means that this information fusion is invalid. $m\left(\theta_{1}\right)$ means the occupied reliability evaluating function. $m\left(\theta_{2}\right)$ means the vacant reliability evaluating function. $m\left(\theta_{1} \cap \theta_{2}\right)$ is the conflict factor's reliability evaluating function, which means the conflicting situation of occupy or empty. $m\left(\theta_{1} \cup \theta_{2}\right)$ means the uncertain reliability evaluating function.

The proportional conflict redistribution rule (PCR) is adopted to fuse the basic reliability of many information got by info source, and the syncretic reliability calculation can be simplified as:

$$
\begin{aligned}
m_{P C R}\left(\theta_{1}\right)= & m_{1}\left(\theta_{1}\right) m_{2}\left(\theta_{1}\right)+m_{1}\left(\theta_{1}\right) m_{2}\left(\theta_{1} \cup \theta_{2}\right)+m_{1}\left(\theta_{1} \cup \theta_{2}\right) m_{2}\left(\theta_{1}\right) \\
& +\frac{m_{1}\left(\theta_{1}\right)^{2} m_{2}\left(\theta_{2}\right)}{m_{1}\left(\theta_{1}\right)+m_{2}\left(\theta_{2}\right)}+\frac{m_{2}\left(\theta_{1}\right)^{2} m_{1}\left(\theta_{2}\right)}{m_{2}\left(\theta_{1}\right)+m_{1}\left(\theta_{2}\right)} . \\
m_{P C R}\left(\theta_{2}\right)= & m_{1}\left(\theta_{2}\right) m_{2}\left(\theta_{2}\right)+m_{1}\left(\theta_{2}\right) m_{2}\left(\theta_{1} \cup \theta_{2}\right)+m_{1}\left(\theta_{1} \cup \theta_{2}\right) m_{2}\left(\theta_{2}\right) \\
& +\frac{m_{1}\left(\theta_{2}\right)^{2} m_{2}\left(\theta_{1}\right)}{m_{1}\left(\theta_{2}\right)+m_{2}\left(\theta_{1}\right)}+\frac{m_{2}\left(\theta_{2}\right)^{2} m_{1}\left(\theta_{1}\right)}{m_{2}\left(\theta_{2}\right)+m_{1}\left(\theta_{1}\right)} .
\end{aligned}
$$

\section{THE EXPERIMENT RESULT AND ANALYSE}

Leader 1-DX mobile robot platform (shown as Fig. 5) is developed by our lab, which equips laser sensor and Point Grey Research's Bumblebee ${ }^{\circledR 2}$ Stereo vision camera systems. Laser sensor is used to avoid barriers. Vision camera systems can collect indoor environmental information abundantly. Fig. (5b) is a typical building in our school which has three floors. Several regions are divided in every storey, and three kinds of artificial labels are pasted on selected positions. Three kinds of experiments have been done in corridors, suites and some typical local areas.

\subsection{The Building Experiment of Global Semantic Topol- ogy Map in the Corridor}

The robot moves in a corridor shown as Fig. (5b). The start is stairway ostium, the corresponding node number is 1 . Based on the leading of artificial signpost labels, the robot goes through 2 6 nodes in order, finally back to the starting node. The No. 1 node has been pastedl-type signpost label, No. 2,4,5 nodes have been pasted II-type signpost labels. 


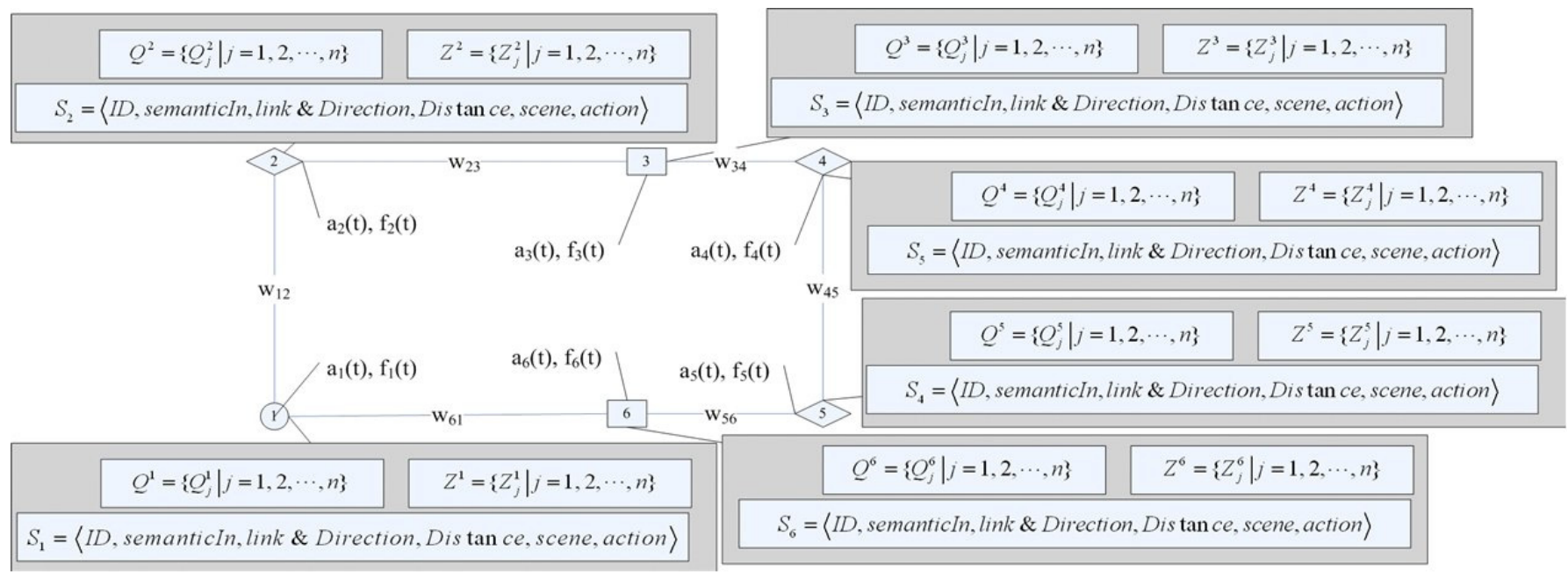

Fig. (6). The mapping process in the corridor of the building.

No. 3 and 6 nodes have been pasted flat labels. The data written in the three kinds of labels are shown in Fig. (6).

\subsection{The Building Experiment of Regional Semantic Plan- ning Map}

The experiment is done in a quasi structured simulant home environmental shown in Fig. (7a). Artificial object labels are pasted on TV cabinets, sink, stove, beds and other items.

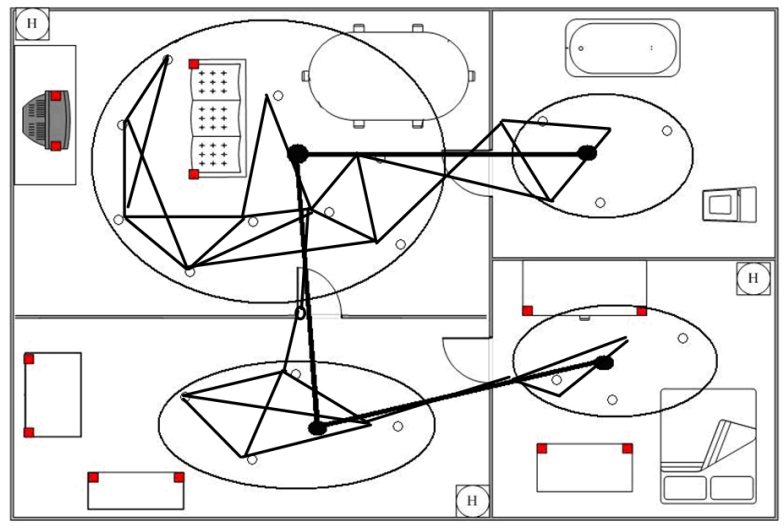

(a) stimulant home environment

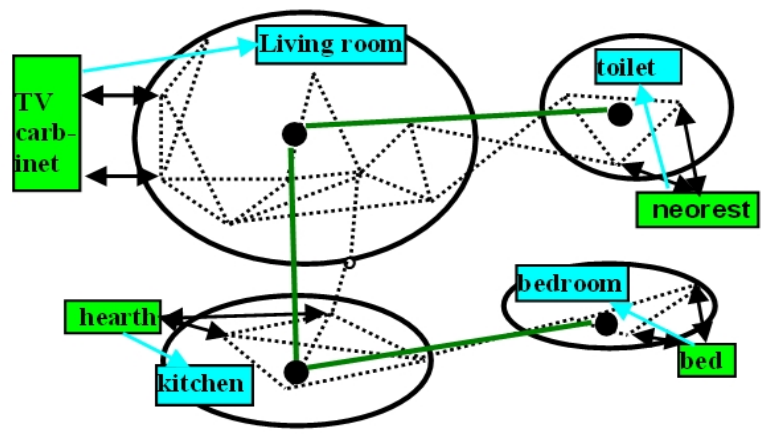

(b) regional semantic map

Fig. (7). Global semantic planning map building.

Image data is real-time collected by the robot. Figs. (8a and $\mathbf{8 b}$ ) are two images collected at different observation points. The label pattern collected from image based on the method of artificial label recognition is shown in Figs. (8c and 8d). QR code is read from (c) and (d). As the QR code information in (c) and (d) are both "TV cabinet," the two results are consistent.

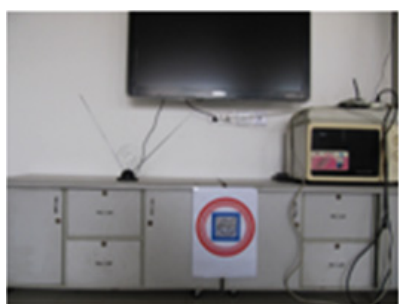

(a)

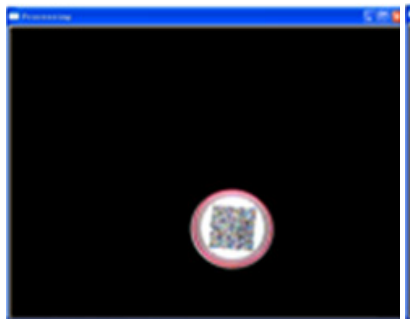

(c)

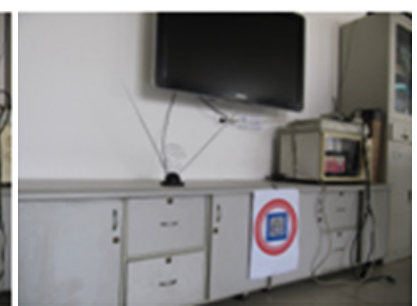

(b)

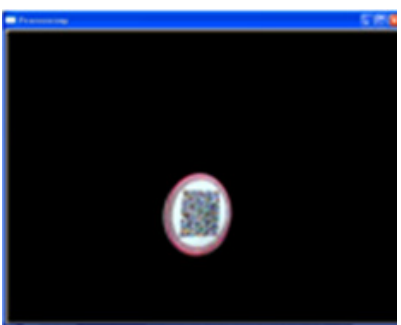

(d)
Fig. (8). Identification and reading of the artificial object label.

The robot moves as the velocity of $0.3 \mathrm{~m} / \mathrm{s}$. The robot starts at arbitrary position and explores the surrounding. A lot of dots shown in Fig. (7a) represent all observation points got by the robot.

Undirected weight graph is built in the end of exploration, as the dotted lines shown in Fig. (7b). The nodes in the undirected weight graph are the observed positions stayed by the robot. The relative graph shows the passing paths for the robot when it does service task. Simultaneously the attributive relations between objects and nodes are obtained (black two-way arrows shown in Fig. (7b)). Four separate sets (the four thick solid ellipses in Fig. (7b)) represent four rooms. The global topological map is built which is partly described by the thick solid green line in Fig. (7b). According to the attributive relations between nodes and objects, we can determine the attributive relations between objects and room, 


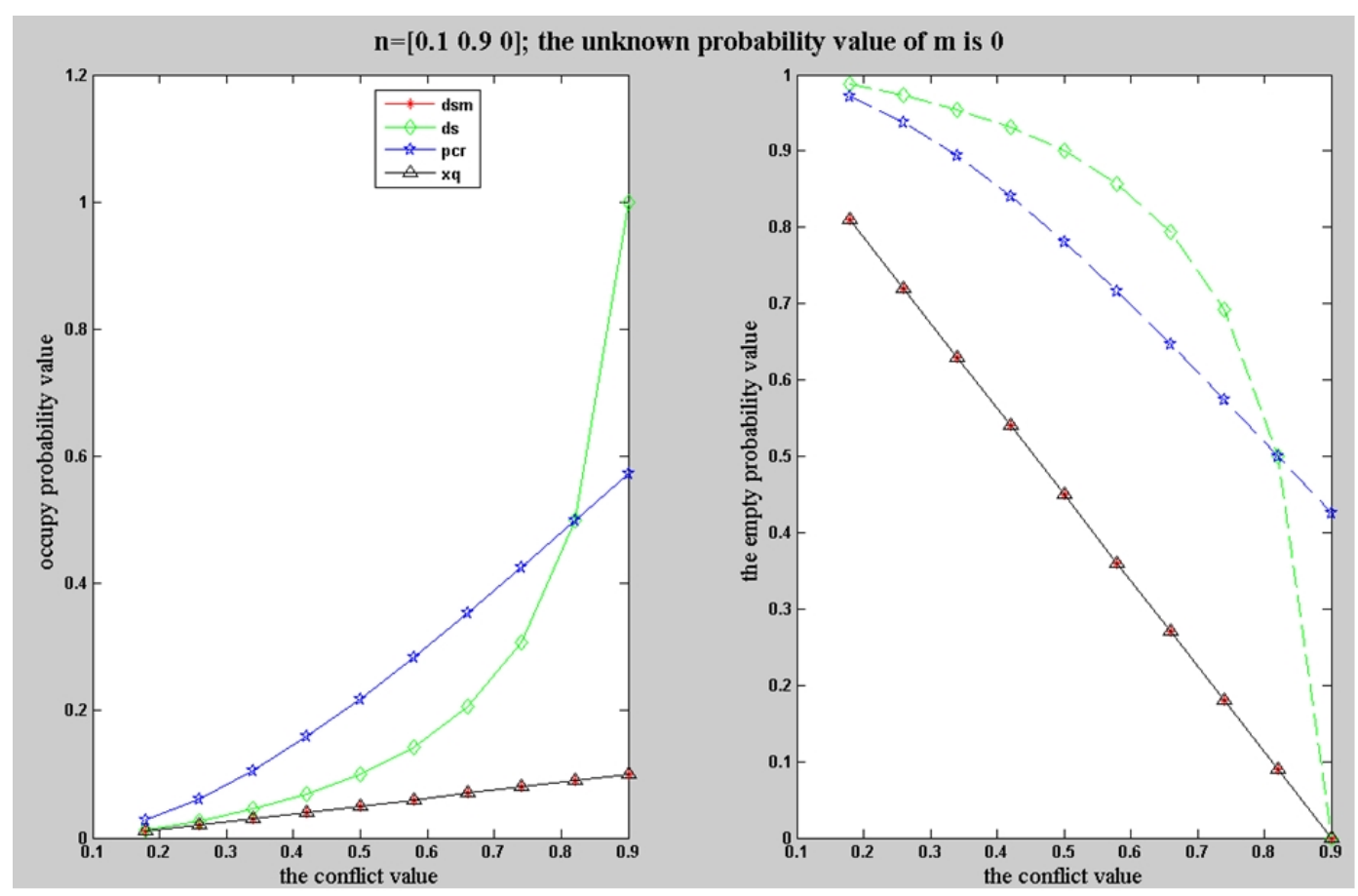

Fig. (9). The fusing result analysis as high conflict.
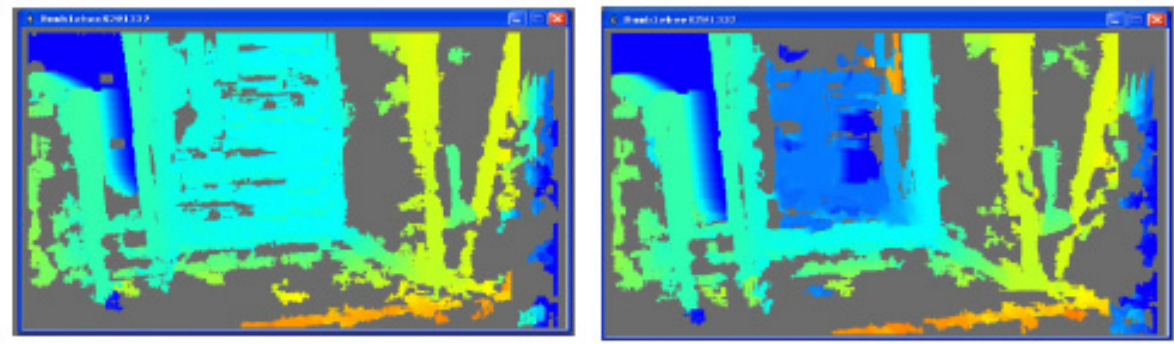

(a) The door is closed (b) the door is opened

Fig. (10) The matching result and map building result in two scenes.

and then determine the functional properties of the room. Up to now, the robot obtains a series of results which include local topology graph (undirected weight graph), regional semantic planning map with room segmentation, the topology relations of nodes, attributive relations between objects, nodes and rooms, the functional property of room, and so on.

\subsection{The Building Experiment of Local Space 3D Map}

Suppose $n=[0.1,0.9,0]$ at time $t$, that means the unknown belief value is 0 . Suppose the unknown value at time $t+1$ is 0 . The occupying belief value increases and the empty value decreases, but their sum is 1 . Then 10 cases come into being shown as below which form the matrix $m$.

$$
m^{T}=\left[\begin{array}{cccccccccc}
0.1 & 0.2 & 0.3 & 0.4 & 0.5 & 0.6 & 0.7 & 0.8 & 0.9 & 1 \\
0.9 & 0.8 & 0.7 & 0.6 & 0.5 & 0.4 & 0.3 & 0.2 & 0.1 & 0 \\
0 & 0 & 0 & 0 & 0 & 0 & 0 & 0 & 0 & 0
\end{array}\right]
$$

The fusion result of the four kinds of fusion rules are shown as Fig. (9).

As shown in Fig. (9), while abnegating process for all conflict, the fusion result of XQ rule deviates from the basic fusion law. The higher the conflict, the greater the bias value, or even wrong fusion result. The DSM rule is better than XQ rule, which is close to the fusion law as less conflict and lapses from the fusion law as biggish conflict. The fusing outcomes of DS and PCR rules essentially accord with the fusion law. When the conflict is small, the effect of the two fusion algorithm is the same. As the conflict higher, the PCR rule more accords with the fusion law, however DS rule has a little windage. As the conflict reaches to the highest value, DS rule's fuses result is wrong.

Fig. (10) showed the building results of 3D description map. When the door is closed, doorsill and door are in the same plane. However, the deep information at the doorsill is different from other features around it when the door is opened. Using this 3D description map, the doorsill is easy to be detected and the robot can use corresponding strategy to get across the door.

\section{CONCLUSION}

According to the service features of the building robot, imitating the ambient cognitive model of the human, three 
hierarchy space model is found for building environment based on the localization of multi-information fusion in intelligent space. Artificial signpost labels are pasted in wide area environment such as corridors, which give navigation information for robots. Artificial object labels are pasted on large objects in the flatlet, which give the information of objects such as their function, property and ascription relation. The simulation and experiment results in three kinds of typical environments showed the effectiveness of the algorithm. Some problems should be studied deeply, that is, the realtime of the algorithm and the complication of the space should be thought for building three hierarchy space model.

\section{CONFLICT OF INTEREST}

The author confirms that this article content has no conflict of interest.

\section{ACKNOWLEDGEMENTS}

This work has partly been supported by National Youth Foundation under Grant 61203330, Shandong Nature Science Foundation under Grant ZR2012FM031, China Postdoctoral Science Foundation funded project 2013M540546, and Shandong Postdoctoral Innovation Foundation 201203058, Shandong major research plan Project 2015GGX103034, Jinan Science and Technology plan Project 201502194.

\section{REFERENCES}

[1] L. Qu, and H. Wang, "An overview of Robot SLAM problem", In: International Conference on Consumer Electronics, Communications and Networks (CECNet), pp. 1953-1956, 2011.
[2] L. D. L. Perera, and N. Eric, "The Simultaneous Localization and Mapping problem in a nonlinear parameter identifiability perspective", The 8th World Congress on Intelligent Control and Automation (WCICA), pp. 630-637, 2010

[3] X. Chen, Y. Y. Han, and Y. Wei, "Map Building Algorithm with High-precision Measurement Based on UFastSLAM", AISS, vol. 4, no. 13, pp. 40-46, 2012.

[4] G. Dissanayake, S. Huang, Z. Wang, and R. Ranasinghe, "A review of recent developments in Simultaneous Localization and Mapping", In: The 6th IEEE International Conference on Industrial and Information Systems (ICIIS), pp. 477-482, 2011.

[5] Y. Guo, H. Jia, Q. Liu, S. Lin, and E. Sun, "Research on Robot SLAM Based on Linear Feature Environment", AISS, vol. 5, no. 3, pp. 252-260, 2013.

[6] B. Patrick, M. Joseph, and K. Benjamin, "The mapping problem: Mobile robot map-building in the hybrid spatial semantic hierarchy", International Journal of Robotics Research, vol. 29, no. 4, pp. 428-459, 2010.

[7] D. Meger, P. Forssén, K. Lai, S. Helmer, S. McCann, T. Southey, M. Baumann, J. J. Little, D. G. Lowe, and C. George, "An attentive semantic robot", Robotics and Autonomous Systems, vol. 56, no. 11, pp. 503-511, 2008.

[8] S. Vasudevan, V. Nguyen, and R. Siegwart, "Towards a Cognitive Probabilistic Representation of Space for Mobile Robots", In: proceedings of the 2006 IEEE International Conference on Information Acquisition (ICIA), pp. 353-359, 2006

[9] Y. Tao, Y. Xia, T. Xu, and X. Chi, "Research Progress of the Scale Invariant Feature Transform (SIFT) Descriptors", JCIT, vol. 5, no. 1, pp. 116-121, 2010.

[10] D. Anguelov, D. Koller, E. Parker, and S. Thrun, "Detecting and Modeling Doors with Mobile Robots", In: proceedings of the International Conference on Robotics and Automation (ICRA), pp. 37773784, 2004.

[11] G. H. Tian, H. T. Jiang, Y. H. Xue, and R. K. Li, "The Design, Recognition, Localization and Application of a New Artificial Landmark," Journal of Shandong University, vol. 41, no. 2, pp. $107-113,2011$.

\footnotetext{
Received: June 10, 2015

(C) Hao et al.; Licensee Bentham Open.
}

Revised: July 29, 2015

Accepted: August 15, 2015

This is an open access article licensed under the terms of the (https://creativecommons.org/licenses/by/4.0/legalcode), which permits unrestricted, noncommercial use, distribution and reproduction in any medium, provided the work is properly cited. 